\title{
ON THE PROPER STEENROD HOMOTOPY GROUPS, AND PROPER EMBEDDINGS OF PLANES INTO 3-MANIFOLDS
}

BY

\author{
MATTHEW G. BRIN ${ }^{1}$ AND T. L. THICKSTUN
}

\begin{abstract}
Standard algebraic invariants of proper homotopy type are discussed. These do not naturally fit into long exact sequences. Groups of proper homotopy classes of proper maps of Euclidean spaces and open annuli which do naturally form a long exact sequence are defined, and a diagram relating these groups to the standard algebraic invariants of proper homotopy type is given. The structures defined are used to compare several notions of essentiality for proper maps. Some results and examples are given for proper maps of spaces into manifolds of dimension 2 and 3. These results are used to add information to a theorem of Brown and Feustel about properly embedding planes in 3-manifolds.
\end{abstract}

1. Introduction. The starting point for these investigations is the following theorem of Brown and Feustel [BF]:

If there is a proper essential map from $E^{2}$ (Euclidean 2-space) into a 3-manifold $M$, then there is a proper essential embedding of $E^{2}$ into $M$. (A map is proper if the inverse image of each compact set is compact.)

The definition of essential as used in [BF] will be given in the next paragraph. We are interested to know which forms of essentiality can be used that make the above theorem true. We expand this question to a study of the relationships between different forms of essentiality. The analysis, restricted to maps of planes into 3-manifolds, allows us finally to write down a stronger version of Brown and Feustel's theorem for a restricted class of 3-manifolds. We will now be more specific.

Let $M$ be a noncompact 3-manifold, let $K$ be a compact set in $M$, let $A$ be a component of $M-K$, and let $G$ be a normal subgroup of $\pi_{1}(A)$. We say that a proper map $f: E^{2} \rightarrow M$ is $(K, G)$-essential if for each disk $D \subseteq E^{2}$ with $f^{-1}(K) \subseteq D$, we have that $f \mid \partial D$ represents a conjugacy class of $\pi_{1}(A)$ outside of $G$. The full statement of the main theorem in $[\mathbf{B F}]$ is:

(1.1) If $M$ is a noncompact 3-manifold, $K \subseteq M$ is compact, $A$ is a component of $M-K, G$ is normal in $\pi_{1}(A)$, and there is a proper map $f: E^{2} \rightarrow M$ that is $(K, G)$-essential, then there is a proper embedding $g: E^{2} \rightarrow M$ that is $(K, G)$-essential.

Received by the editors July $30,1984$.

1980 Mathematics Subject Classification. Primary 57N65; Secondary 57N10, 57N05.

Key words and phrases. Proper map, proper homotopy type, Steenrod homotopy group, noncompact manifold.

' Partially supported by Title F and UAC grants from SUNY and the SUNY Foundation. 
Let $f: X \rightarrow Y$ be proper. We say that $f$ is discretely trivial if for every compact $K$ in $Y$ there is a compact $C$ in $X$ and a map $f^{\prime}: X \rightarrow Y$ so that $\operatorname{Im}\left(f^{\prime}\right)$ misses $K$ and $f^{\prime}=f$ off $C$. Note that a proper map $f: E^{2} \rightarrow M$ fails to be $(K, G)$-essential for all pairs $(K, G)$ if and only if it is discretely trivial. We say that a proper map $f: X \rightarrow Y$ is compactly trivial if for every compact $K$ in $Y$ there is a proper homotopy $H$ : $X \times I \rightarrow Y$ (the homotopy $H$ is a proper homotopy if it is a proper map on $X \times I$ ) so that $H_{0}=f$ and $\operatorname{Im}\left(H_{1}\right)$ misses $K$. Note that for reasonable domains (e.g., locally finite simplicial complexes) compactly trivial implies discretely trivial. Lastly we say that a proper map $f: X \rightarrow Y$ is properly trivial if there is a proper map $F$ : $X \times[0, \infty) \rightarrow Y$ so that $F(x, 0)=f(x)$ for all $x \in X$. Clearly properly trivial implies compactly trivial. The following questions arise where $X$ and $Y$ are locally finite simplicial complexes and $f: X \rightarrow Y$ is proper.

(1.2) If $f$ is discretely trivial, must it be compactly trivial?

(1.3) If $f$ is compactly trivial, must it be properly trivial?

The more delicate question is (1.3). The following summarizes the status of (1.3) in those cases that we answer.

(a) $X$ is arbitrary, $\quad Y=2$-manifold "yes";

(b) $X=S^{2}$, $\quad Y=3$-manifold "yes";

(c) $X=S^{1}, E^{1}, E^{2}, \quad Y=3$-manifold "no".

The problem originally considered in connection with the theorem of Brown and Feustel concerned $X=E^{2}$ and $Y$ a 3-manifold. In this case we find conditions on $Y$ for which the answer is "yes". A corollary of our findings (see (4.7) below) is:

(1.5) If $M$ is a 3-manifold with $\pi_{2} M=0$ and $f: E^{2} \rightarrow M$ is proper, then the following are equivalent:

(i) $f$ is discretely trivial.

(ii) $f$ is compactly trivial.

(iii) $f$ is properly trivial.

Together with (1.1) this implies:

(1.6) If $M$ is a 3-manifold with $\pi_{2} M=0$ and $f: E^{2} \rightarrow M$ is proper and not properly trivial, then there is a proper embedding $g: E^{2} \rightarrow M$ that is not discretely trivial.

In $\$ 2$ of this paper we describe in some detail the algebraic framework into which these questions fit. (Question (1.3) must be modified slightly for compact domains to take care of a "basepoint" problem.) The structures defined consist of groups calculated from the usual homotopy groups, other groups (the Steenrod homotopy groups of the title), and various homomorphisms and exact sequences connecting them. Our contribution here is primarily arrangement (see especially (2.13) below) since much of $\$ 2$ is gathered from work in other areas: The algebraic topology of compact metric spaces and inverse limits [S, Mil1, Mil2, BK]; proper homotopy theory [B1, B2, Ce, D]; and "strong" shape theory [Q1, Q2, F, KO]. The bridge between the first two topics was first indicated in [Ch]. A very abstract combined view of all three topics was written out in $[\mathbf{E H}]$. Much of $\$ 2$ below is contained 
implicitly in [EH] although the authors of the present paper do not know a practical way of extracting $\$ 2$ from $[\mathbf{E H}]$. We include many details for completeness since they are scattered in the literature or are otherwise unrecorded, and omit many others that can be easily supplied by the reader.

In $\S 3$ we show how the material in $\S 2$ applies to questions (1.2) and (1.3). In $\S 4$ we give the results and counterexamples for low-dimensional manifolds that are summarized in (1.4).

We will use $Z, Z_{+}, E^{i}$ and $S^{i}$ to represent, respectively, the integers, the nonnegative integers, Euclidean $i$-space and the $i$-sphere. All homology groups will be over $Z$. In $\$ 4$ we consider manifolds and maps between manifolds. These will all be piecewise linear. We will use Fr $X$ for $\bar{X} \cap(\overline{Y-X})$ (the topological frontier of $X$ ) when $X \subseteq Y$, and $\partial X$ for the combinatorial boundary of $X$ when $X$ is a manifold.

The authors would like to thank Ross Geoghegan for many useful conversations. The first author would like to thank Southwest Texas State University and Institut des Hautes Études Scientifiques for their hospitality and use of facilities during the period from June 1982 to June 1983 when part of this research was done.

2. All spaces in this section are locally finite $\mathrm{CW}$ complexes. With proper map and proper homotopy as defined above, one defines proper homotopy equivalence and proper homotopy type in the obvious way. All the structures that we define in this section are invariants of proper homotopy type.

An end of $X$ is an equivalence class of sequences $\left(U_{i}\right)$ of nonempty, connected open sets in $X$ with empty intersection so that $U_{i+1} \subseteq U_{i}$, each $U_{i}$ has noncompact closure and compact frontier and where $\left(U_{i}\right) \sim\left(V_{i}\right)$ if the two sequences are cofinal (i.e., every $i$ has a $j$ and every $j$ has a $k$ so that $U_{k} \subseteq V_{j} \subseteq U_{i}$ ). There is another definition equivalent to this that is also useful. An end of $X$ is an equivalence class of proper maps $r:[0, \infty) \rightarrow X$ where $r_{1} \sim r_{2}$ if $r_{1} \mid Z_{+}$and $r_{2} \mid Z_{+}$are properly homotopic. Compact spaces have no ends, $E^{1}$ has 2 ends (denoted $+\infty$ and $-\infty$ ) and $E^{n}, n \geqslant 2$, and $[0, \infty$ ) have one end each (denoted $\infty$ ). If $f: X \rightarrow Y$ is proper and $e$ is an end of $X$, then $f$ carries $e$ to a unique end of $Y$ which we denote by $f(e)$. If $e$ is an end of $X$, then a base ray for $e$ is a proper map $r:[0, \infty) \rightarrow X$ so that $r(\infty)=e$ (or equivalently $[r]=e$ ). A base ray for $e$ plays a role analogous to a basepoint.

Let $e$ be an end of a connected space $X$ represented by $\left(U_{j}\right)$ and let $r$ be a base ray for $e$ chosen so that $r[j, \infty) \subseteq U_{j}$ for each $j \in Z_{+}$. Consider the following diagram whose rightmost terms are $\pi_{0}(X)=[*]$ :

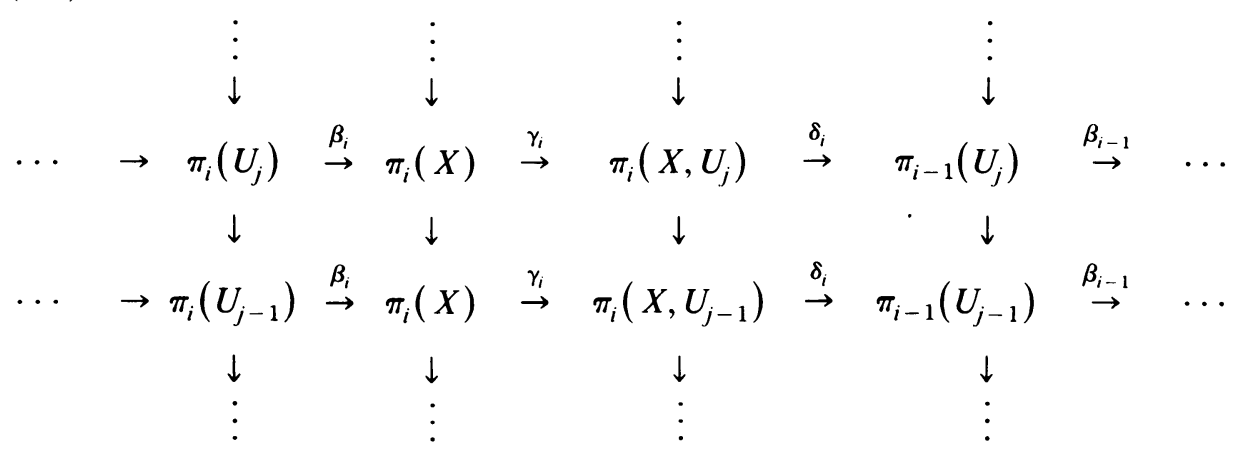


The groups in row $j$ are based at $r(j)$. Each horizontal row is the homotopy exact sequence of a pair. The vertical arrows between rows $j$ and $j-1$ are the inclusion induced homomorphisms composed with the change of basepoint isomorphisms using the path $r[j-1, j]$. The diagram commutes.

Each column is an inverse sequence of groups which depends on $\left(U_{j}\right)$ and $r$. Replacing $\left(U_{j}\right)$ by a sequence $\left(V_{j}\right)$ that also represents $e$ gives a sequence closely related to the first. Let $\left(A_{i}, \phi_{i}\right)$ and $\left(B_{i}, \theta_{i}\right)$ be inverse sequences of groups. We say that these sequences are pro-isomorphic (p.i.) if homomorphisms $\left(\alpha_{i}\right)$ exist making the following diagram commute:

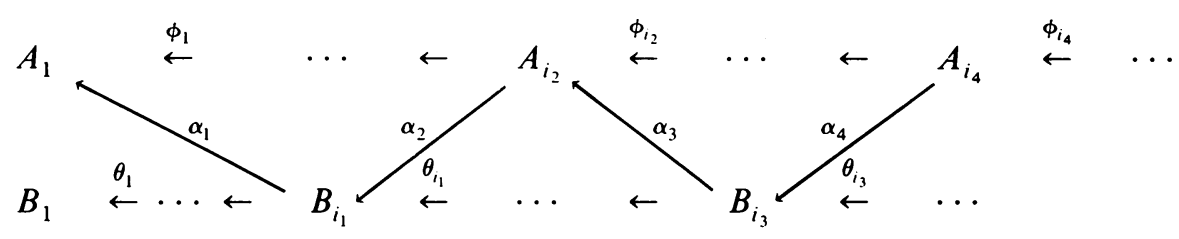

We require $1<i_{1}<i_{2}<\cdots$. If a column in (2.1) is altered by replacing $\left(U_{j}\right)$ by $\left(V_{j}\right)$ with $\left(U_{j}\right) \sim\left(V_{j}\right)$, then the resulting inverse sequence of groups is p.i. to the original. One can check that the homomorphisms $\alpha_{i}$ in (2.2) induce inverse isomorphisms between $\lim _{\leftarrow} A_{i}$ and $\lim _{\leftarrow} B_{i}$. A change of base ray is more drastic. An example of Siebenmann is the following subset of $E^{3}$ :

$$
\begin{aligned}
X= & \left\{(x, y, z) \mid x^{2}+y^{2}=1, z \geqslant 0\right\} \cup\{(2,0, z) \mid z \geqslant 0\} \\
& \cup\left\{(x, 0, z) \mid 1 \leqslant x \leqslant 2, z \in Z_{+}\right\} .
\end{aligned}
$$

For this space, the column of (2.1) whose entries are $\pi_{1}\left(U_{j}\right)$ will have inverse limit $Z$ if base ray $r(t)=(1,0, t)$ is used and trivial inverse limit if $r(t)=(2,0, t)$ is used. All algebraic objects in this section will depend on a fixed base ray, but for brevity this will not be expressed in the notation.

By taking the inverse limit of each column, (2.1) yields the following sequence:

$$
\cdots \rightarrow \lim _{\leftarrow} \pi_{i}\left(U_{j}\right) \stackrel{\beta_{i}^{\prime}}{\rightarrow} \pi_{i}(X) \stackrel{\gamma_{i}^{\prime}}{\rightarrow} \lim \pi_{i}\left(X, U_{j}\right) \stackrel{\delta_{i}^{\prime}}{\rightarrow} \underset{\leftarrow}{\lim } \pi_{i-1}\left(U_{j}\right) \rightarrow \cdots .
$$

In general this sequence fails to be exact, but is a chain complex. It is exact at $\lim _{\leftarrow} \pi_{i}\left(U_{j}\right)$ for all $i \geqslant 1$, which the reader may show now directly or wait until later when it follows from more general considerations.

There is a sequence closely related to (2.4) which is exact. Let $*$ be a basepoint for $S^{i}$ and let $s:[0, \infty) \rightarrow S^{i} \times[0, \infty)$ take $t$ to $(*, t)$. Let $\pi_{i}^{S}(e), i \geqslant 0$, be equivalence classes of proper maps $f: S^{i} \times[0, \infty) \rightarrow X$ satisfying $f \circ s=r$. For $i=0$ we further require that $f\left(e_{1}\right)=f\left(e_{2}\right)=e$ where $e_{1}$ and $e_{2}$ are the two ends of $S^{0} \times[0, \infty)$. We say $f \sim g$ if $f$ and $g$ are properly homotopic rel $\{*\} \times[0, \infty)$. A product $f g$ is defined in $\pi_{i}^{S}(e)$ for $i \geqslant 1$ by forming the product

$$
\left(f \mid S^{i} \times\{t\}\right)\left(g \mid S^{i} \times\{t\}\right)
$$


as in $\pi_{i}(X ; r(t))$ at each $t$. The set $\pi_{0}^{S}(e)$ is pointed by letting $f: S^{0} \times[0, \infty) \rightarrow X$ taking $(x, t)$ to $r(t)$ represent the distinguished element. There is a natural map $\alpha_{i}$ : $\pi_{i}{ }^{S}(e) \rightarrow \lim _{\leftarrow} \pi_{i}\left(U_{j}\right)$ taking $f$ to the sequence $\left(\left[f \mid S^{i} \times\left\{t_{j}\right\}\right]^{\prime}\right)$ where $t_{1}<t_{2}<\cdots$ are chosen so that $f\left(S^{i} \times\left[t_{j}, \infty\right)\right) \subseteq U_{j}$ for each $j$ and $\left[f \mid S^{i} \times\left\{t_{j}\right\}\right]^{\prime}$ represents the image in $\pi_{i}\left(U_{j} ; r(j)\right)$ of $\left[f \mid S^{i} \times\left\{t_{j}\right\}\right] \in \pi_{i}\left(U_{j} ; r\left(t_{j}\right)\right)$ under the change of basepoint isomorphism using the path $\left.r \| j, t_{j}\right]$. The reader can show that the map is well defined and an epimorphism. We defer the discussion of kernel $\alpha_{i}$.

Now let $E^{i}$ for $i \geqslant 1$, be represented by spherical coordinates $(t, x), t \in[0, \infty)$, $x \in S^{i-1}$, where all pairs $(0, x)$ represent the origin. Here let $s:[0, \infty) \rightarrow E^{i}$ take $t$ to $(t, *)$. Let $\pi_{i}^{S}(X, e)$ be equivalence classes of proper maps $f: E^{i} \rightarrow X$ satisfying $f \circ s=r$. For $i=1$ we require that $f(-\infty)=f(\infty)=e$. We say $f \sim g$ if $f$ and $g$ are properly homotopic rel $\operatorname{Im}(s)$. A product $f g$ is defined on $\pi_{i}^{S}(X, e)$ for $i \geqslant 2$ by forming

$$
\left(f \mid S_{t}^{i-1}\right)\left(g \mid S_{t}^{i-1}\right)
$$

as in $\pi_{i-1}(X ; r(t))$ at each $t$, where $S_{t}^{i-1}$ is the set of all $(t, x)$ for a fixed $t$. The set $\pi_{1}^{S}(X, e)$ is pointed by letting $f: E^{1} \rightarrow X$ taking $(t, x)$ to $r(t)$ represent the distinguished element. There is a natural map

$$
\varepsilon_{i}: \pi_{i}^{S}(X, e) \rightarrow \lim _{\leftarrow} \pi_{i}\left(X, U_{j}\right)
$$

taking $f$ to the sequence $\left(\left[f \mid(t, x): t \leqslant t_{j}\right]^{\prime}\right)$ where $t_{1}<t_{2}<\cdots$ are chosen so that for each $j f(t, x) \subseteq U_{j}$ if $t \geqslant t_{j}$ and where $\left[f \mid(t, x): t \leqslant t_{j}\right]^{\prime}$ represents the image in $\pi_{i}\left(X, U_{j} ; r(j)\right)$ under the change of basepoint isomorphism using $\left.r \| j, t_{j}\right]$ of the element $\left[f \mid(t, x): t \leqslant t_{j}\right]$ in $\pi_{i}\left(X, U_{j} ; r\left(t_{j}\right)\right]$. Again the reader should show that $\varepsilon_{i}$ is well defined and an epimorphism. Again discussion of $\operatorname{ker} \varepsilon_{i}$ is deferred. We will see in the next section that $\operatorname{ker} \varepsilon_{i}$ is trivial if $\operatorname{ker} \alpha_{i-1}$ is trivial.

We call the groups $\pi_{i}^{S}(e)$ and $\pi_{i}^{S}(X, e)$, respectively, the absolute and relative Steenrod homotopy groups of $e$.

We have a sequence ending at $\pi_{0}(X)$

$$
\cdots \rightarrow \pi_{i}^{S}(e) \stackrel{\beta_{i}^{\prime \prime}}{\rightarrow} \pi_{i}(X) \stackrel{\gamma_{i}^{\prime \prime}}{\rightarrow} \pi_{i}^{S}(X, e) \stackrel{\delta_{i}^{\prime \prime}}{\rightarrow} \pi_{i-1}^{S}(e) \rightarrow \cdots
$$

where the fact that each $\pi_{i}^{S}$ is based at $r$ and each $\pi_{i}$ is based at $r(0)$ is suppressed from the notation. To help define the homomorphisms, we let $q: B^{i} \rightarrow S^{i}$ be the quotient map from the unit ball in $E^{i}$ onto $S^{i}$ whose only singularity is to take all of $\partial B^{i}$ to $\{*\}$. Now $\beta_{i}^{\prime \prime}[f]$ is $f\left|S^{i} \times\{0\}, \gamma_{i}^{\prime \prime}[f]\right| B^{i}$ is $f \circ q$ while $\gamma_{i}^{\prime \prime}[f](t, x)$ is $r(t-1)$ for $t \geqslant 1$, and $\delta_{i}^{\prime \prime}[f](x, t)$ is $f(t, x)$ again using spherical coordinates for $E^{i}$. Exactness is straightforward where, in the rightmost terms without group structures, exact means "kernel = image" and "kernel" is the inverse image of the distinguished element. The sequence (2.5) is an analog of the homotopy exact sequence of the pair $(M, \partial M)$ for a compact manifold with boundary where $e$ plays the role of a missing boundary component. In fact, if $F$ is a component of $\partial M, e$ is the end of $M-F$, and $r$ is a base ray for $e$ following the product structure in a collar on $F$, then there are natural homomorphisms from $\pi_{i}(F)$ to $\pi_{i}^{S}(e)$ and from $\pi_{i}(M, F)$ to 
$\pi_{i}^{S}(M-F, e)$. These do not in general seem to be onto, but a straightforward exercise shows them to be one-to-one.

The relation between (2.5) and (2.4), the failure of exactness of (2.4) and the nature of $\operatorname{ker} \alpha_{i}$ and $\operatorname{ker} \varepsilon_{i}$ can be discussed after another invariant of inverse sequences of groups is defined. Let $\left(A_{i}, \phi_{i}\right)$ be an inverse sequence of groups and let $\Pi A_{i}$ act on itself on the left by defining

$$
\left(x_{i}\right)\left(g_{i}\right)=\left(x_{i} g_{i}\left(\phi_{i} x_{i+1}\right)^{-1}\right) \text {. }
$$

We let $\lim ^{1} A_{i}$ denote the orbit space of this action. The set $\lim ^{1} A_{i}$ inherits a group structure from $\Pi A_{i}$ if the groups $A_{i}$ are abelian. Otherwise $\lim ^{1} A_{i}$ is a pointed set with the orbit of $(1,1, \ldots)$ as the distinguished element. We have $\lim ^{1} A_{i}$ trivial (one point) if $d: \Pi A_{i} \rightarrow \Pi A_{i}$ defined by

$$
d\left(x_{i}\right)=\left(x_{i}\left(\phi_{i} x_{i+1}\right)^{-1}\right)
$$

is onto. Another criterion exists for the triviality of $\lim ^{1} A_{i}$ if each $A_{i}$ is countable. Let $A_{i}^{k}$ denote $\operatorname{Im}\left(A_{i+k}\right)$ in $A_{i}$ under $\phi_{i} \circ \cdots \circ \phi_{i+k-1}$. We say that $\left(A_{i}, \phi_{i}\right)$ is semistable (ss) if for each $i$ there is a $K$ so that $k>K$ implies $A_{i}^{k}=A_{i}^{K}$. A sequence is ss if and only if it is p.i. to a sequence where all the bonding maps are epimorphisms. (A sequence is stable if it is p.i. to a sequence where all the bonding maps are isomorphisms.) For inverse sequences of arbitrary groups, ss implies the triviality of $\lim ^{1}$. For sequences of countable groups the converse is true (see [Gr] for the abelian case and [Ge] for the general). The following holds.

(2.6) A short exact sequence of inverse sequences of groups

$$
\{1\} \rightarrow\left(A_{i}, \phi_{i}\right) \rightarrow\left(B_{i}, \theta_{i}\right) \rightarrow\left(C_{i}, \psi_{i}\right) \rightarrow\{1\}
$$

gives rise to a natural sequence of groups and pointed sets in the nonabelian case, and groups in the abelian case

$$
\begin{aligned}
\{1\} & \rightarrow \underset{\leftarrow}{\lim A_{i}} \rightarrow \underset{\leftarrow}{\lim B_{i}} \rightarrow \underset{\leftarrow}{\lim C_{i}} \\
& \stackrel{\Delta}{\rightarrow} \lim ^{1} A_{i} \rightarrow \lim ^{1} B_{i} \rightarrow \lim ^{1} C_{i} \rightarrow\{*\}
\end{aligned}
$$

which is exact in the sense that

(i) "kernel = image" at all six positions, and

(ii) the map $\Delta$ extends to a natural action of $\lim _{\leftarrow} C_{i}$ on $\lim ^{1} A_{i}$ such that elements of $\lim ^{1} A_{i}$ are in the same orbit if and only if they have the same image in $\lim ^{1} B_{i}$.

This is [BK, Proposition 2.3, Chapter 9] where no proof is given. A proof in the abelian case is in [Mas, Appendix]. Here we content ourselves with defining $\Delta$. The rest of the proof is long but straightforward. Let $\left(c_{i}\right) \in \underset{\leftarrow}{\lim } C_{i}$ and let $\left(b_{i}\right) \in \Pi B_{i}$ map onto $\left(c_{i}\right)$. Then $d\left(b_{i}\right)=\left(b_{i}\left(\theta_{i} b_{i+1}\right)^{-1}\right)$ maps to $(1,1, \ldots)$ in $\lim C_{i}$ and thus can be regarded as an element in $\Pi A_{i}$ and also in $\lim ^{1} A_{i}$. If $\left(b_{i}^{\prime}\right) \in \overleftarrow{\Pi B_{i}}$ also maps onto $\left(c_{i}\right)$, then the action of $\left(b_{i}^{\prime} b_{i}^{-1}\right)$ on $d\left(b_{i}\right)$ gives

$$
\left(b_{i}^{\prime} b_{i}^{-1} b_{i}\left(\theta_{i} b_{i+1}\right)^{-1}\left(\theta_{i} b_{i+1}^{\prime} b_{i+1}^{-1}\right)^{-1}\right)=d\left(b_{i}^{\prime}\right) .
$$

Thus setting $\Delta\left(c_{i}\right)=d\left(b_{i}\right)$ gives a well-defined map. 
The sequence (2.7) says that $\lim$ preserves kernels and, in the abelian case, $\lim ^{1}$ preserves cokernels. (To say that $\leftarrow_{\text {imm }}^{1}$ preserves images is wrong.)

We now consider

$$
\alpha_{i}: \pi_{i}^{S}(e) \rightarrow \lim _{\leftarrow} \pi_{i}\left(U_{j}\right) \text { and } \varepsilon_{i}: \pi_{i}^{S}(X, e) \rightarrow \underset{\leftarrow}{\lim } \pi_{i}\left(X, U_{j}\right) .
$$

The following are exact:

$$
\begin{gathered}
\{1\} \rightarrow \lim ^{1} \pi_{i+1}\left(U_{j}\right) \rightarrow \pi_{i}^{S}(e) \stackrel{\alpha_{i}}{\rightarrow} \underset{\leftarrow}{\lim \pi_{i}}\left(U_{j}\right) \rightarrow\{1\}, \quad i \geqslant 0, \\
\{1\} \rightarrow \lim ^{1} \pi_{i+1}\left(X, U_{j}\right) \rightarrow \pi_{i}^{S}(X, e) \stackrel{\varepsilon_{i}}{\rightarrow} \underset{\leftarrow}{\lim } \pi_{i}\left(X, U_{j}\right) \rightarrow\{1\}, \quad i \geqslant 2,
\end{gathered}
$$

and

$$
\{*\} \rightarrow \lim ^{1}\left(\operatorname{ker} \beta_{1}\right) \rightarrow \pi_{1}^{S}(X, e) \stackrel{e_{1}}{\rightarrow} \underset{\leftarrow}{\lim } \pi_{1}\left(X, U_{j}\right) \rightarrow\{*\},
$$

where (2.8) for $i=0$ and (2.10) are exact as sequences of pointed sets. (The validity of (2.8) for $i=0$ is proved in [Mih].)

Before giving arguments, we show that (2.9) will follow from

$$
\{1\} \rightarrow \lim ^{1}\left(\operatorname{ker} \beta_{i}\right) \rightarrow \pi_{i}^{S}(X, e) \stackrel{\varepsilon_{i}}{\rightarrow} \lim _{\leftarrow} \pi_{i}\left(X, U_{j}\right) \rightarrow\{1\}, \quad i \geqslant 2 .
$$

Consider the short exact sequence

$$
\{1\} \rightarrow\left(\operatorname{ker} \delta_{i+1}\right) \rightarrow\left(\pi_{i+1}\left(X, U_{j}\right)\right) \stackrel{\delta_{i+1}}{\rightarrow}\left(\operatorname{Im} \delta_{i+1}\right) \rightarrow\{1\}
$$

which gives by (2.6) the exact sequence

$$
\begin{aligned}
\{1\} & \rightarrow \underset{\leftarrow}{\lim \left(\operatorname{ker} \delta_{i+1}\right)} \rightarrow \underset{\leftarrow}{\lim \pi_{i+1}\left(X, U_{j}\right) \rightarrow} \underset{\leftarrow}{\lim \left(\operatorname{Im} \delta_{i+1}\right)} \\
& \rightarrow \lim ^{1}\left(\operatorname{ker} \delta_{i+1}\right) \rightarrow \lim ^{1} \pi_{i+1}\left(X, U_{j}\right) \stackrel{\mu}{\rightarrow} \lim ^{1}\left(\operatorname{Im} \delta_{i+1}\right) \rightarrow\{*\} .
\end{aligned}
$$

But $\operatorname{Im} \delta_{i+1}=\operatorname{ker} \beta_{i}$ and $\operatorname{ker} \delta_{i+1}=\operatorname{Im} \gamma_{i+1}$. However $\lim ^{1}\left(\operatorname{Im} \gamma_{i+1}\right)$ must be trivial since $\left(\operatorname{Im} \gamma_{i+1}\right)$ is the homomorphic image of the stable sequence $\pi_{i+1}(X)$ (note that $i+1$ is not the running index for the sequence) and $\lim ^{1}$ preserves cokernels. For $i \geqslant 2,(2.12)$ is a sequence of groups giving

$$
\lim ^{1} \pi_{i+1}\left(X, U_{j}\right) \cong \lim ^{1}\left(\operatorname{ker} \beta_{i}\right) .
$$

For $i=1, \mu^{-1}(*)=(*)$ but we cannot conclude that $\mu$ is one-to-one.

To verify (2.8) we will define a map $k$ from $\operatorname{ker} \alpha_{i}$ to $\lim ^{1} \pi_{i+1}\left(U_{j}\right)$ which must then be shown to be a homomorphism, one-to-one and onto for $i \geqslant 1$, and just one-to-one and onto for $i=0$. If $f: S^{i} \times[0, \infty) \rightarrow X$ represents an element in ker $\alpha_{i}$, then it can be reparametrized so that, for each $j, f\left(S^{i} \times[j, \infty)\right) \subseteq U_{j}$ and $f \mid S^{i} \times\{j\}$ extends to a singular map of an $(i+1)$-ball into $U_{j}$. For ease in notation we will refer to this extension as $B_{j}$. If $j<k$, we will let $B_{j} B_{k}$ represent the singular sphere obtained by sewing together the maps $B_{j}, f \mid S^{i} \times[j, k]$ and $-B_{k}$, where the minus sign indicates a reversal of orientation needed to insure that the equation $B_{j} B_{k}+B_{k} B_{l}=B_{j} B_{l}$ makes sense if sufficient care is paid to basepoints. For $j>k$ we reverse the 
orientation of the annular section. We regard $B_{j} B_{k}$ as based at $(*, j), * \in S^{i}$. To view it in $\pi_{i}\left(U_{l}\right), l \leqslant j$, then a change of basepoint isomorphism using $r[[l, j]$ is used. We now let $k[f]$ be the class of the sequence $\left(B_{j} B_{j+1}\right)$ in $\lim ^{1} \pi_{i+1}\left(U_{j}\right)$. If balls $B_{j}^{\prime}$ are chosen instead of $B_{j}$, then it is seen that the action of $\left(B_{j}^{\prime} B_{j}\right)$ on $\left(B_{j} B_{j+1}\right)$ yields $\left(B_{j}^{\prime} B_{j+1}^{\prime}\right)$. Independence of reparametrization is easy, and if $f^{\prime}$ is properly homotopic to $f$ rel $\{*\} \times[0, \infty)$, then each can be reparametrized so that the track of $S^{i} \times[j, \infty)$ stays in $U_{j}$ during the homotopy. The sequence of spheres for $f$ and $f^{\prime}$ are thus equivalent. To check that $k$ is a homomorphism when $i \geqslant 1$ is straightforward. To show that $k$ is one-to-one and onto, one starts with the trivial annulus $f: S^{i} \times[0, \infty)$ defined by $f(x, t)=r(t)$ and pipes spheres at regular intervals to the annulus to show onto, and to the trivial spanning $(i+1)$-balls at each $r(j)$ to show one-to-one while reviewing the definitions. To show one-to-one for $i=0$ one pipes circles to the spanning arcs connecting the two rays of arbitrary elements of $\pi_{0}^{S}(e)$.

To deal with (2.10) and (2.11) we again put $E^{i}$ in spherical coordinates $(t, x)$, $t \in[0, \infty), x \in S^{i-1}$. If $f: E^{i} \rightarrow X$ represents an element in ker $\varepsilon_{i}$, then reparametrization gives $f(t, x) \in U_{j}$ whenever $t \geqslant j$. If $C_{j}=\{(x, t) \mid t \leqslant j\}$, then $f \mid C_{j}$ is homotopic rel $\partial C_{j}$ to a singular map $B_{j}$ into $U_{j}$. Now the spheres $B_{j} B_{j+1}$ (notation of previous paragraph) are null homotopic in $X$ and are thus elements in the kernel of $\beta_{i}: \pi_{i}\left(U_{j}\right) \rightarrow \pi_{i}(X)$. The rest of the argument is indentical to the previous one.

We now fit together a diagram ending with the terms $\pi_{0}(X)$ :

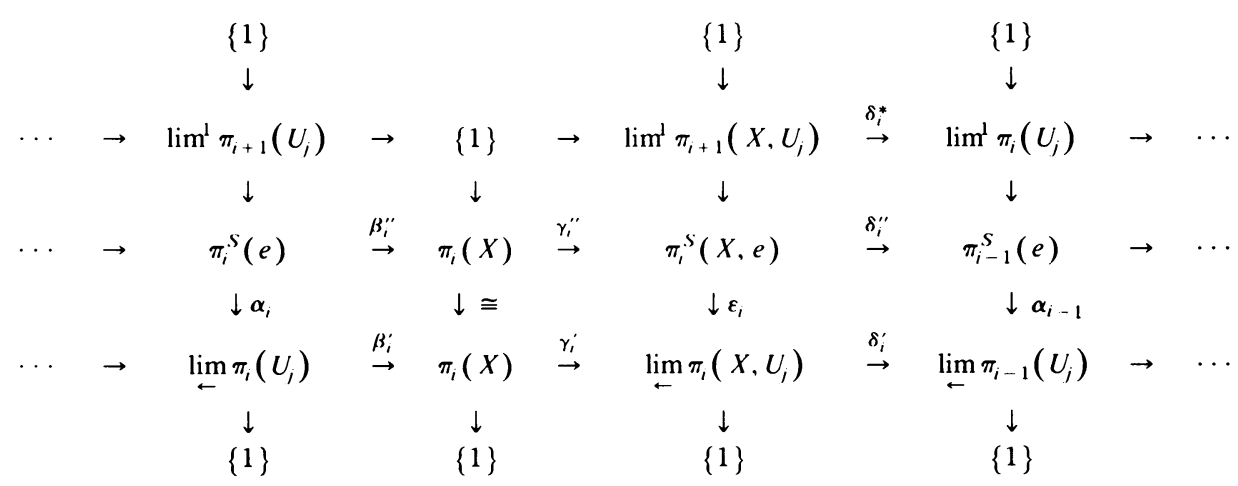

In the diagram, $\lim ^{1} \pi_{2}\left(X, U_{j}\right)$ is replaced by $\lim ^{1}\left(\operatorname{ker} \beta_{1}\right)$. Each column is exact and the middle row is exact. The ladder between the bottom two rows commutes by the construction of the groups and the homomorphisms between them. The map $\delta_{i}^{*}$ is induced by $\delta_{i}^{\prime \prime}$ and it exists because the square involving $\delta_{i}^{\prime \prime}$ and $\delta_{i}^{\prime}$ commutes. For $i \geqslant 2$ it is easily checked that $\delta_{i}^{*}$ is also the homomorphism between $\lim ^{1}$ groups induced by the inverse sequence morphism $\delta_{i}$. For $i \geqslant 2$ this is the homomorphism $\mu$ of (2.12) composed with the homomorphism $\eta$ of

$$
\begin{aligned}
\{1\} & \rightarrow \underset{\leftarrow}{\lim \left(\operatorname{ker} \beta_{i}\right)} \rightarrow \underset{\leftarrow}{\lim \pi_{i}}\left(U_{j}\right) \rightarrow \underset{\leftarrow}{\lim \left(\operatorname{Im} \beta_{i}\right)} \\
& \rightarrow \lim ^{1}\left(\operatorname{ker} \beta_{i}\right) \stackrel{\eta}{\rightarrow} \lim ^{1} \pi_{i}\left(U_{j}\right) \rightarrow \lim ^{1}\left(\operatorname{Im} \beta_{i}\right) \rightarrow\{1\} .
\end{aligned}
$$


When $i=1$, the map $\delta_{1}^{*}$ is simply the map $\eta$. It is seen that the ladder between the top two rows commutes by some simple diagram chasing and, in the few cases where the sets involved are not groups, by direct checking. There are six entries in (2.13) that are groups but are not necessarily abelian. In these positions it is easily seen that the image of the arrow from the left is a normal subgroup.

We can now discuss a measure of the failure of exactness of (2.4) or the bottom row of (2.13). Assume a diagram of groups, not necessarily abelian

$$
\begin{array}{cccccccccc}
\ldots & \rightarrow & A_{i+1} & \rightarrow & A_{i} & \rightarrow & A_{i-1} & \rightarrow & A_{i-2} \\
& & \downarrow & & \downarrow & & \downarrow & & \downarrow \\
\cdots & \rightarrow & B_{i+1} & \rightarrow & B_{i} & \rightarrow & B_{i-1} & \rightarrow & B_{i-2} \\
& & \downarrow & & \downarrow & & \downarrow & & \\
\cdots & & \rightarrow & C_{i+1} & \rightarrow & C_{i} & \rightarrow & C_{i-1} & &
\end{array}
$$

where each row is a chain complex in which each image is a normal subgroup, where the upper vertical arrows are monomorphisms, the lower vertical arrows are epimorphisms, and each column is exact. We can define homology groups in the usual way down to $H_{i}(C), H_{i-1}(A)$ and $H_{i-1}(B)$. The standard derivation of the long exact homology sequence from a short exact sequence of chain complexes goes through unaltered in the situation above to give a sequence of homology groups

$$
\cdots \rightarrow H_{i}(A) \rightarrow H_{i}(B) \rightarrow H_{i}(C) \rightarrow H_{i-1}(A) \rightarrow H_{i-1}(B)
$$

which is exact down to the term $H_{i-1}(A)$. We can truncate (2.13) so that its last few columns are:

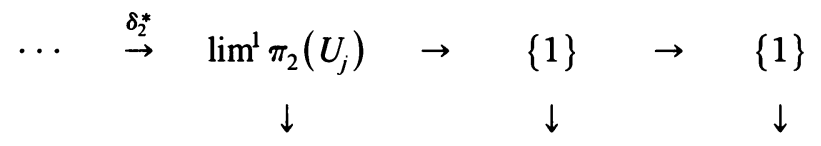

$$
\begin{aligned}
& \ldots \quad \stackrel{\delta_{2}^{\prime \prime}}{\rightarrow} \quad \pi_{1}^{S}(e) \quad \stackrel{\beta_{1}^{\prime \prime}}{\rightarrow} \pi_{1}(X) \stackrel{\gamma_{1}^{\prime \prime}}{\rightarrow} \operatorname{Im} \gamma_{1}^{\prime \prime} \\
& \begin{array}{cccc} 
& \downarrow \alpha_{1} & & \downarrow \cong \\
\cdots & \stackrel{\delta_{2}^{\prime}}{\rightarrow} & \lim _{\leftarrow} \pi_{1}\left(U_{j}\right) \stackrel{\beta_{1}^{\prime}}{\rightarrow} & \pi_{1}(X)
\end{array}
\end{aligned}
$$

Since the middle row is exact, every third term in the long exact sequence (2.16) is trivial and the remaining terms are isomorphic in pairs. This says that $\operatorname{ker} \beta_{i}^{\prime}=$ $\operatorname{Im} \delta_{i+1}^{\prime}$ for $i \geqslant 1$ (the previously announced statement that (2.4) is exact at the terms $\left.\lim _{\leftarrow} \pi_{i}\left(U_{j}\right)\right)$ and that

$$
\left(\operatorname{ker} \gamma_{i}^{\prime} / \operatorname{Im} \beta_{i}^{\prime}\right) \cong \operatorname{ker} \delta_{i}^{*} \quad \text { for } i \geqslant 2 \text {, }
$$

and

$$
\left(\operatorname{ker} \delta_{i}^{\prime} / \operatorname{Im} \gamma_{i}^{\prime}\right) \cong \operatorname{coker} \delta_{i}^{*} \quad \text { for } i \geqslant 2 .
$$

The statements to replace (2.18) and (2.19) when $i=1$ are

$$
\operatorname{ker} \gamma_{1}^{\prime}=\operatorname{Im} \beta_{1}^{\prime} \text { if and only if } \operatorname{ker} \delta_{1}^{*}=\{*\}
$$

and

$$
\gamma_{1}^{\prime} \text { is onto if and only if } \delta_{1}^{*} \text { is onto. }
$$


Statement (2.21) is the correct replacement for (2.19) since $\underset{\leftarrow}{\lim } \pi_{0}\left(U_{j}\right)$ contains only one element. These two statements must be checked separately. To show (2.20) one must show the existence of a well-defined function $m$ : $\operatorname{ker} \gamma_{1}^{\prime} \rightarrow \operatorname{ker} \delta_{1}^{*}$ that is onto so that $m(f)=m(g)$ if and only if $[f][g]^{-1}$ is in $\operatorname{Im} \beta_{1}^{\prime}$. To show (2.21) one must show the existence of a multiple valued (set) function $n: \lim _{\leftarrow} \pi_{1}\left(X, U_{j}\right) \rightarrow \lim ^{1} \pi_{1}\left(U_{j}\right)$ so that $y, y^{\prime}$ in $n(x)$ and $y$ in $\operatorname{Im} \delta_{1}^{*}$ implies $y^{\prime}$ in $\operatorname{Im} \delta_{1}^{*}$, and so that $x$ is in $\operatorname{Im} \gamma_{1}^{\prime}$ if and only if some $y$ in $n(x)$ is in $\operatorname{Im} \delta_{1}^{*}$. We leave the details to the reader. We do not know whether there is a natural bijection between $\operatorname{ker} \gamma_{1}^{\prime} / \operatorname{Im} \beta_{1}^{\prime}$ and $\operatorname{ker} \delta_{1}^{*}$ or whether the apparently multiple valued $n$ above is actually single valued.

(2.22) EXAMPLE. We can give a very simple example to show that $(2.4)$ generally fails to be exact at $\lim _{\leftarrow} \pi_{i}\left(X, U_{j}\right), i \geqslant 1$. Let $E^{i+1}, i \geqslant 1$, again be represented in spherical coordinates $(t, x)$ and let $M^{i+1}$ be $E^{i+1}$ with open balls of radius $\frac{1}{4}$ and centered at all $(j, *), j \in Z_{+}$, removed. By abusing notation slightly we can identify $\delta_{i}^{*}$ with the function $\eta$ in (2.14). The exactness of (2.14) says (even for $i=1$ ) that $\delta_{i}^{*}$ is onto if and only if $\lim ^{1}\left(\operatorname{Im} \beta_{i}\right)$ is trivial or, equivalently, the sequence $\left(\operatorname{Im} \beta_{i}\right)$ is semistable. It is easy to check that $\left(\operatorname{Im} \beta_{i}\right)$ is not semistable for $M^{i+1}$. The reader must wait until $\S 4$ for an example showing the failure of exactness of $(2.4)$ at $\pi_{i}(X)$.

REMARKS. We point out that (2.18) and (2.19) follow from much more general considerations. Let

$$
\left(A_{i j} ; \theta_{i j}: A_{i j} \rightarrow A_{i, j-1} ; \phi_{i j}: A_{i+1, j} \rightarrow A_{i, j}\right), \quad j \in Z, i \geqslant 0,
$$

be an exact sequence of inverse sequences of abelian groups in that, for fixed $i$, $\left(A_{i j}, \theta_{i j}\right)$ is exact and, for fixed $j,\left(A_{i j}, \phi_{i j}\right)$ is an inverse sequence. Then $\left(\lim _{\leftarrow} A_{i j}, \phi_{i j}^{\prime}\right)$ and $\left(\lim _{i}^{1} A_{i j}, \phi_{i j}^{*}\right)$ are chain complexes with homology groups $H_{j}^{\prime}(A)$ and $H_{j}^{\prime \prime}(A)$, respectively. Using (2.7), a straightforward argument shows

$$
H_{j}^{\prime}(A) \cong H_{j+2}^{\prime \prime}(A) \text {. }
$$

The functor $\lim ^{1}$ on inverse sequences is the first right derived functor of the left exact, covariant functor lim. For inverse sequences (or, more generally, inverse systems over countable indexing sets as opposed to arbitrary indexing sets), the higher derived functors of lim vanish (see [BK, Chapter XI, §6.4] for remarks and references). Thus (2.7) follows from general considerations about derived functors (see [Mac, Chapter XII, §9 and HS, Chapter IV, §6]). The isomorphism in (2.23) can also be derived from the definition of derived functor. The isomorphism in (2.23) also holds for other inexact functors whose second derived functors vanish (e.g., Hom and $\otimes$ on abelian groups) where the index increase of 2 is replaced by a decrease of 2 depending on the variance and whether the functor is right or left exact. In light of these comparisons, (2.8) and (2.9) can be viewed as "universal coefficient theorems".

3. In this section we discuss (1.2) and (1.3) in light of the structures developed in $\S 2$. Since the structures of $\$ 2$ depend heavily on a given base ray, we will introduce some restrictions. These restrictions will have greater effect on maps with compact domains. 
If $f: K \rightarrow X$ is a proper map between infinite, locally finite, simplicial complexes, then we will assume that base rays $r_{1}:[0, \infty) \rightarrow K$ and $r_{2}:[0, \infty) \rightarrow X$ are given, where $r_{1}$ is an embedding to a subcomplex and $r_{2}=f \circ r_{1}$. In this situation we will say that $f$ is proper rel the given base ray $r_{2}$. Note that a proper map is always rel some base ray. If $K$ is finite, then we can form the wedge of $K$ and $[0, \infty)$ at $\{0\}$ and require that $f[0, \infty)$ be a given base ray of $X$. We then say that the extended $f$ is proper rel the given base ray. We will abuse notation and let $K$ represent both the original finite complex and the wedge with $[0, \infty)$ and we will let $f$ represent both the original map and the extension. Context will determine a given reference. In this section we require all maps to be rel a given base ray.

We also restrict homotopies. If $r:[0, \infty) \rightarrow X$ is a base ray and $F: K \times J \rightarrow X$ is a homotopy $(J=[0, n]$ or $[0, \infty))$ so that $F_{0}$ carries a basepoint $x_{0} \in K$ to $r(0)$, then we require that $F\left(x_{0}, t\right)=r(t)$ for $t \in J$. This is not a severe restriction. Let $f, g$ : $K \rightarrow X$ be proper rel $r:[0, \infty) \rightarrow X$ and let $F: K \times I \rightarrow X$ and $G: K \times[0, \infty) \rightarrow X$ be proper extensions of $f$ and $g$ in that $F_{0}=f$ and $G_{0}=g$. Let $L$ be a compact subset of $X$ and assume that $F_{1}$ misses $L$ and $\left.r \| n, \infty\right)$ misses $L$. If $x_{0} \in K$ is the start of the given base ray in $K$, then there are proper extensions $F^{\prime}: K \times[0, n] \rightarrow X$ and $G^{\prime}$ : $K \times[0, \infty) \rightarrow X$ of $f$ and $g$ so that $\operatorname{Im} F_{n}^{\prime}$ misses $L$ and so that $F^{\prime}\left(x_{0}, t\right)=r(t)$ for $t \in[0, n]$ and $G^{\prime}\left(x_{0}, t\right)=r(t)$ for $t \in[0, \infty)$.

Note that if $f: K \rightarrow X$ is proper rel some base ray and $K=S^{i}$, then we can regard [ $f$ ] as an element of $\pi_{i}(X)$ flexibly taken to be any of the groups labeled $\pi_{i}(X)$ in (2.1) and (2.13). If $K=E^{i}$, then we can regard [ $f$ ] as an element of $\pi_{i}^{S}(X, e)$ where $e$ is the end corresponding to the given base ray. The notation in the statements in this section is consistent with that of (2.1) and (2.13).

In (3.1)-(3.8) we assume an $f: K \rightarrow X$ that is proper rel some base ray. The demonstrations are either trivial or straightforward and are left to the reader.

(3.1) If $K$ is finite, then $f$ is discretely trivial.

(3.2) If $K=S^{i}$, then $f$ is compactly trivial if and only if $[f]$ is in $\operatorname{ker} \gamma_{i}^{\prime}$.

(3.3) If $K=S^{i}$, then $f$ is properly trivial if and only if $[f]$ is in $\operatorname{Im} \beta_{i}^{\prime}$.

(3.4) The following are equivalent:

(a) Every $f: S^{i} \rightarrow X$ proper rel $r$ that is compactly trivial is properly trivial.

(b) $\operatorname{Im} \beta_{i}^{\prime}=\operatorname{ker} \gamma_{i}^{\prime}$.

(c) $\operatorname{ker} \delta_{i}^{*}$ is trivial.

An example that shows that (a), (b), and (c) of (3.4) need not hold is given in the next section. The reader can easily supply examples to illustrate various possibilities relating to (3.2) and (3.3).

(3.5) If $K=E^{i}$, then $f$ is discretely trivial if and only if $[f]$ is in $\operatorname{ker}\left(\delta_{i}^{\prime} \varepsilon_{i}\right)$.

(3.6) If $K=E^{i}$, then $f$ is compactly trivial if and only if $[f]$ is in ker $\varepsilon_{i}$.

(3.7) If $K=E^{i}$, then $f$ is properly trivial if and only if $[f]$ is trivial in $\pi_{i}^{S}(X, e)$.

(3.8) Every $f: E^{i} \rightarrow X$ proper rel $r$

(a) is discretely trivial if and only if $\operatorname{ker} \beta_{i-1}^{\prime}$ is trivial;

(b) that is discretely trivial is compactly trivial if and only if $\operatorname{ker} \delta_{i}^{\prime}$ is trivial;

(c) that is compactly trivial is properly trivial if and only if $\operatorname{ker} \varepsilon_{i}$ is trivial.

In the rest of this section we discuss Question (1.3) for $f: K \rightarrow X$ proper rel $r$ for an arbitrary locally finite complex $K$. We discuss this under assumptions of triviality 
of the kernels of the homomorphisms $\left(\alpha_{i}\right)$. These turn out to be very strong assumptions. What we obtain mirrors various movability results in shape theory. We will several times, without reference, use the Geoghegan-Gray equivalence between the semistability of an inverse sequence of countable groups and the triviality of its $\lim ^{1}$

(3.9) If $\operatorname{ker} \alpha_{i-1}$ is trivial, then $\operatorname{ker} \varepsilon_{i}$ is trivial.

This follows from $\operatorname{ker} \varepsilon_{i}=\lim ^{1}\left(\operatorname{ker} \beta_{i}\right), \operatorname{ker} \alpha_{i-1}=\lim ^{1}\left(\pi_{i}\left(U_{j}\right)\right)$ and from

(3.10) If $\theta:\left(A_{i}\right) \rightarrow\left(B_{i}\right)$ is a homomorphism of inverse sequences $\left(\theta=\left(\theta_{i}: A_{i} \rightarrow B_{i}\right)\right.$ and ladder commutes), the bonding homomorphisms of $\left(B_{i}\right)$ are monomorphisms and $\left(A_{i}\right)$ is semistable, then $\left(\operatorname{ker} \theta_{i}\right)$ is semistable.

(3.11) If $\operatorname{ker} \alpha_{i-1}$ is trivial, then for each $k$ there exists a $J \geqslant k$ so that if $f: E^{i} \rightarrow X$ represents an element of $\operatorname{ker}\left(\delta_{i}^{\prime} \varepsilon_{i}\right)$ in $\pi_{i}^{S}(X, e)$ and $f$ properly homotopes to $f^{\prime}$ : $E^{i} \rightarrow U_{J}$, then $f^{\prime}$ is properly trivial in $\bar{U}_{k}$.

Proof. Since $\left(\pi_{i}\left(U_{j}\right)\right)$ is semistable, there is a $J \geqslant k$ so that $\left.\operatorname{Im} \pi_{i}\left(U_{j}\right)\right)$, for any $j \geqslant J$, in $\pi_{i}\left(U_{k}\right)$ is the same as $\operatorname{Im} \pi_{i}\left(U_{J}\right)$ in $\pi_{i}\left(U_{k}\right)$. Let $f$ properly homotope to $f^{\prime}$ : $E^{i} \rightarrow U_{J}$. We can assume that the homotopy is relative to the complement of some compact subset of $E^{i}$. Now consider (2.13) with $X$ replaced by $\bar{U}_{k}$ or $\bar{U}_{J}$. The system $\left(U_{j}\right)$ for either new diagram is obtained from the old by dropping a finite number of terms. Thus we have $\lim ^{1}\left(\pi_{i}\left(U_{j}\right)\right)$ trivial, $\alpha_{i-1}$ and $\varepsilon_{i}$ one-to-one, and [ $f^{\prime}$ ] an element of $\operatorname{ker}\left(\delta_{i}^{\prime} \varepsilon_{i}\right)$ in either diagram. Thus $\left[f^{\prime}\right]$ is in $\operatorname{ker} \delta_{i}^{\prime \prime}=\operatorname{Im} \gamma_{i}^{\prime \prime}$ and $f^{\prime}$ is properly homotopic in $\bar{U}_{J}$ to a "sphere with tail". Since the "tail" is a section of the base ray, our choice of $U_{J}$ says that $f^{\prime}$ properly homotopes in $\bar{U}_{k}$ to a map into any $U_{j}, j \geqslant J$. Thus in (2.13) with $\bar{U}_{k}$ in the role of $X,\left[f^{\prime}\right]$ is an element in ker $\varepsilon_{i}$ and is thus trivial in $\pi_{i}^{S}\left(\bar{U}_{k}, e\right)$.

This gives a sufficient condition for a positive answer to (1.3).

(3.12) Let $K$ be locally finite and finite dimensional with top dimension $p$ and let $f$ : $K \rightarrow X$ be proper rel $r$. If for all $i \leqslant p$ we have $\lim ^{1}\left(\pi_{i}\left(U_{j}\right)\right)$ trivial and $f$ is compactly trivial, then $f$ is properly trivial.

We leave the details of the proof to the reader. Use (3.11) repeatedly $p$ times, together with the fact that pushing the $i$-skeleton of $K$ to $\infty$ stretches the interior of each $(i+1)$-simplex in $K$ into a proper map of $E^{i+1}$. The finite-dimensionality of $K$ and the compactly trivial hypothesis allow enough room for the repetitions.

We have a minor modification of (3.12) whose proof we leave to the reader.

(3.13) Let $K$ be locally finite and finite dimensional with top dimension $p$ and let $f$ : $K \rightarrow X$ be proper rel $r$. Assume $K$ is $q$-connected for some $q<p$ and that $\lim ^{1}\left(\pi_{i}\left(U_{j}\right)\right)$ is trivial for all $i$ with $q<i \leqslant p$. Then $f$ is properly trivial if it is compactly trivial.

4. In this section we give results and examples with low-dimensional manifolds. We first need a fact that gives information about $\pi_{i}^{S}(X, e)$ similar to that given in (2.10) and (2.11). Notation is as in $\$ 2$.

(4.1) Let $f: E^{i} \rightarrow X$ be proper rel $r$ and compactly trivial. Assume each $\beta_{i}: \pi_{i}\left(U_{j}\right) \rightarrow$ $\pi_{i}(X)$ is injective for each $j$. Then $f$ is properly trivial. Further if $f^{\prime}: E^{i} \rightarrow U_{j}$ is properly homotopic to $f$, then $f^{\prime}$ is properly trivial in $\bar{U}_{j}$.

The first conclusion is weaker than (3.8)(c). The second is straightforward and is left to the reader. 
This now allows us to state

(4.2) Let $f: K \rightarrow M$ be proper rel $r$, where $K$ is locally finite and $M$ is a 2-manifold. If $f$ is compactly trivial, then it is properly trivial.

The proof follows from (4.1) in much the same way that (3.12) follows from (3.11). The difference in the treatment of the subscripts between (4.1) and (3.11) allows us to drop the assumption of finite-dimensionality. The only 2-manifold that cannot be arranged so that $\beta_{i}$ is always injective is $E^{2}$, and a map into $E^{2}$ is properly trivial if and only if it can be properly homotoped off some point.

Before we give results about 3-manifolds we list some needed facts.

(4.3) If $M=\cup^{\infty} B_{i}, B_{i} \subseteq \operatorname{Int} B_{i+1}$, and each $B_{i}$ is a homotopy 3-cell, then $M$ is properly homotopy equivalent to $E^{3}$.

(4.4) Let $M$ be a 3-manifold and let $S$ be a finite union of disjoint, embedded, closed, 2-sided surfaces in Int $M$ so that if $F$ is a component of $S$, then $\pi_{1}(F) \rightarrow \pi_{1}(M)$ is one-to-one and $F$ is not a 2-sphere that bounds a homotopy ball in $M$.

(a) If $f: S^{2} \rightarrow(M-S)$ is homotopically trivial in $M$, then it is homotopically trivial in $M-S$.

(b) If $g$ : $S^{2} \times I \rightarrow M$ carries $S^{2} \times\{0,1\}$ into $M-S$ and $g \mid S^{2} \times\{0\}$ is not homotopically trivial in $M$, then either $g \mid S^{2} \times\{0\}$ and $g \mid S^{2} \times\{1\}$ are homotopic in $M-S$, or $g \mid S^{2} \times\{0\}$ homotopes into a 2-sphere or projective plane component of $S$.

The proof of (4.4) proceeds by lifting the homotopy $g$ and the implied homotopy of (a) to the universal cover $\tilde{M}$ of $M$. If $\tilde{S}$ is the preimage of $S$ in $\tilde{M}$, then each component of $\tilde{S}$ is a plane or a nontrivial sphere. Removing undesired components of the preimage of $\tilde{S}$ under the lifted homotopies is straightforward (see [L, Proof of Lemma, p. 124]). The result follows using the isomorphisms between $H_{2}(\tilde{M}), \pi_{2}(\tilde{M})$ and $\pi_{2}(M)$.

(4.5) If $M$ is a 3-manifold and $\left(U_{j}\right)$ are as in $\$ 2$ so that $\pi_{2}\left(U_{j}\right) \rightarrow \pi_{2}(M)$ is trivial for some $j$, then either $M$ is properly homotopy equivalent to $E^{3}$ or $\pi_{2}\left(U_{j}\right)=0$ for all but finitely many $j$.

This follows from the sphere theorem and from (4.3).

(4.6) If $M$ is a 3-manifold, $\left(U_{j}\right)$ are as in $\S 2$, and, for all $j$, each component $F$ of $\operatorname{Fr} U_{j}$ has $\pi_{1}(F) \rightarrow \pi_{1}(M)$ injective, then either $M$ is properly homotopy equivalent to $E^{3}$ or $\pi_{2}\left(U_{j}\right) \rightarrow \pi_{2}(M)$ is injective for all but finitely many $j$.

This follows from (4.3) and (4.4)(a) and from the observation that spheres in $\operatorname{Fr} U_{j}$ can be piped together and to other components (if any) in $\operatorname{Fr} U_{j}$ in $U_{j}$ without destroying the hypothesis. So we may assume that $\operatorname{Fr} U_{j}$ is a 2-sphere or has no 2-spheres for each $j$.

We can now state to what extent the answer to (1.3) is yes for maps of planes into 3-manifolds. Later an example will show that this is "best possible".

(4.7) Let $M$ be a 3-manifold and $r$ be a base ray to some end of $M$. Let $\left(U_{j}\right)$ be as in $\$ 2$ and assume that $\pi_{2}\left(U_{j}\right) \rightarrow \pi_{2}(M)$ is trivial for some $j$ or assume that for each $j$, each component $F$ of $\operatorname{Fr} U_{j}$ has $\pi_{1}(F) \rightarrow \pi_{1}(M)$ injective. Then every $f: E^{2} \rightarrow M$ proper rel $r$ that is compactly trivial is properly trivial.

Proof. By (3.8)(c) we need to show ker $\varepsilon_{2}$ is trivial. But $\operatorname{ker} \varepsilon_{2}$ is $\lim ^{1}\left(\operatorname{ker} \beta_{2}\right)$ where $\beta_{2}:\left(\pi_{2}\left(U_{j}\right) \rightarrow \pi_{2}(M)\right)$ is a homomorphism of inverse sequences induced by inclu- 
sion. By (4.5) and (4.6) either $M$ is properly homotopy equivalent to $E^{3}$ (in which case $\left(\operatorname{ker} \beta_{2}\right)$ is stable) or $\left(\operatorname{ker} \beta_{2}\right)$ is trivial.

We now show that the answer to (1.3) is yes for maps of 2-spheres into 3-manifolds. The proof uses basic 3-manifold techniques and needs no assumption of the map being proper rel some fixed base ray. The proof actually says something about the structure of the manifold. We give some definitions needed in the statement. Let $S^{2} \times[0, \infty)$ be altered by replacing each $S^{2} \times[i, i+1]$ by a connected sum of $S^{2} \times[i, i+1]$ and a finite number of homotopy 3-spheres. We call the resulting space a proper homotopy $S^{2} \times[0, \infty)$ (p.h. $S^{2} \times[0, \infty)$ ). Now let a space $X$ be double covered by a p.h. $S^{2} \times[0, \infty)$ so that each $S^{2} \times\{i\}, i \in\left(Z_{+}\right.$ $-\{0\}$ ), projects to a separating, 2-sided projective plane. We cannot say that $X$ is obtained from $P^{2} \times[0, \infty)$ by taking connected sums with homotopy spheres (see the first paragraph of proof of 10.2 in $[\mathbf{H}]$ ); however, we will still refer to such an $X$ as a p.h. $P^{2} \times[0, \infty)$.

(4.8) Let $M$ be a 3-manifold and let $f: S^{2} \rightarrow M$ be compactly trivial. Then $f$ is properly trivial. Further, if $f$ is not homotopic to a constant in $M$, then there is a separating, embedded 2-sphere or projective plane $\Sigma$ in $M$ so that f homotopes into $\Sigma$ and so that the closure of a complementary domain of $\Sigma$ in $M$ is a p.h. $S^{2} \times[0, \infty)$ or a p.h. $P^{2} \times[0, \infty)$.

Proof. The theorem is true if $f$ is homotopic to a constant so we assume it is not. Write $M=\cup M_{i}$, where each $M_{i}$ is a connected, compact 3-manifold, $M_{i} \subseteq \dot{M}_{i+1}$ and $f\left(S^{2}\right) \subseteq M_{0}$. By adding 1-handles and taking a subsequence we may also assume that each component of $\operatorname{Fr} M_{i}$ separates $M$ for each $i$. For each $i$, there is a homotopy $H^{i}: S^{2} \times I \rightarrow M$ so that $H_{0}^{i}=f$ and $\operatorname{Im} H_{1}^{i}$ misses $M_{i}$. Thus for each integer pair $i \geqslant j$, there is a unique component $F_{i j}$ of $\operatorname{Fr} M_{j}$ separating $\operatorname{Im} H_{0}^{i}$ from $\operatorname{Im} H_{1}^{i}$. For each $j$, there is therefore a component $F_{j}$ of $\operatorname{Fr} M_{j}$ so that $F_{i j}=F_{j}$ for infinitely many $i \geqslant j$. By passing to a subsequence repeatedly we may assume:

(i) For each $i, \operatorname{Im} H_{1}^{i}$ misses $M_{i}$ and is contained in $M_{i+1}$;

(ii) for each $j$, there is a component $F_{j}$ of $\operatorname{Fr} M_{j}$ that separates $\operatorname{Im} H_{0}^{i}$ from $\operatorname{Im} H_{1}^{i}$ whenever $i \geqslant j$.

If we apply the sphere theorem to each $H_{1}^{i}$, we obtain a 2-sphere or 2-sided projective plane $\Sigma_{i}$ embedded in $M_{i+1}-M_{i}$ that is not contractible in $M$. We will show that $f$ homotopes into $\Sigma_{1}$ and that each pair $\Sigma_{i}, \Sigma_{i+1}$ is the boundary of a compact 3-manifold covered by a connected sum of $S^{2} \times I$ and a finite number of homotopy spheres. If $\Sigma_{1}$ fails to separate, then a simple closed curve demonstrating this would have nonzero intersection number with $f$ since $f$ is nontrivial and $\pi_{2}\left(\Sigma_{1}\right) \rightarrow \pi_{2}(M)$ is one-to-one. This would contradict the compact triviality of $f$ and so $\Sigma_{1}$ must separate. These facts will suffice to finish the theorem.

We now give the promised demonstrations. Fix an $i$ and consider $H^{i+2}: S^{2} \times I \rightarrow$ $M$. Let $Y_{i}$ and $Y_{i+1}$ be the components of $M_{i+1}-\stackrel{\circ}{M}_{i}$ and $M_{i+2}-\dot{M}_{i+1}$ containing $\Sigma_{i}$ and $\Sigma_{i+1}$, respectively. There are compact submanifolds $W_{i}$ and $W_{i+1}$ of $S^{2} \times \stackrel{i}{I}$ so that for $j=i, i+1, W_{j}$ is a component of $\left(H^{i+2}\right)^{-1}\left(Y_{j}\right), \partial W_{j}$ contains exactly two components $A_{j}$ and $B_{j}$ that each separate $S^{2} \times\{0\}$ from $S^{2} \times\{1\}$ and so that $H^{i+2}$ carries $A_{j}$ and $B_{j}$ to disjoint components of $\operatorname{Fr} Y_{j}$. Let $\tilde{M}$ be the universal cover of $M$, 
let $\tilde{H}^{i+2}$ be a lift of $H^{i+2}$, for $j=i, i+1$, let $\tilde{Y}_{j}$ be a component of the preimage of $Y_{j}$ so that $\tilde{H}^{i+2}\left(W_{j}\right) \subseteq \tilde{Y}_{j}$, and let $\tilde{\Sigma}_{j}$ be a component of the preimage of $\Sigma_{j}$ in $\tilde{Y}_{j}$.

Now fix $j$ at either $i$ or $i+1$. Let $\tilde{Y}_{j}^{\prime}$ be the union of $\tilde{Y}_{j}$ and all its complementary domains that have compact closure. Let $W_{j}^{\prime}$ be that component of $\left(\tilde{H}^{i+2}\right)^{-1}\left(\tilde{Y}_{j}^{\prime}\right)$ that contains $W_{j}$. We note that $A_{j}$ and $B_{j}$ are components of $\partial W_{j}^{\prime}$ since otherwise $\tilde{H}_{0}^{i+2}$ would be homologous to zero (and thus null homotopic) in $\tilde{M}$. Let $F$ be a component of $\partial W_{j}^{\prime}$ other than $A_{j}$ or $B_{j}$. Since $F$ bounds a compact 3-manifold in $S^{2} \times I, F$ must be carried with degree 0 to a component of $\operatorname{Fr} \tilde{Y}_{j}^{\prime}$ by $\tilde{H}^{i+2}$. The degree of $\tilde{H}^{i+2}$ must be nonzero on both $A_{j}$ and $B_{j}$ since $\tilde{H}_{0}^{i+2}$ is not null homotopic. Since $\tilde{H}^{i+2}\left(A_{j}\right)$ and $\tilde{H}^{i+2}\left(B_{j}\right)$ are disjoint, the total degree of $\tilde{H}^{i+2}$ on $\partial W_{j}^{\prime}$ into Fr $\tilde{Y}_{j}^{\prime}$ must be nonzero and thus the degree of $\tilde{H}^{i+2} \mid W_{j}^{\prime}$ is nonzero. (Use the correspondence between the geometric and algebraic methods of computing degree [E, Lemma 2.1]). In particular $\tilde{H}^{i+2}$ carries $W_{j}^{\prime}$ onto $\tilde{Y}_{j}^{\prime}$ and, more to the point, Im $\tilde{H}^{i+2}$ must contain $\tilde{\Sigma}_{j}$ and Im $H^{i+2}$ must contain $\Sigma_{j}$. Applying (4.4)(b) to $H^{i+2}$ we learn that $H_{0}^{i+2}=f$ must homotope into $\Sigma_{j}$. Applying (4.4)(b) to $\tilde{H}^{i+2}$ we learn that some nonzero multiple (since $\tilde{H}_{0}^{i+2}$ is not null homologous) of $\left[\tilde{\Sigma}_{i}\right]$ in $H_{2}(\tilde{M})$ is homologous to [ $\tilde{\Sigma}_{i+1}$ ] in $H_{2}(\tilde{M})$. Since $H_{2}(\tilde{M})$ is torsion free, we have that $\tilde{\Sigma}_{i}$ and $\tilde{\Sigma}_{i+1}$ bound a compact, necessarily simply connected 3 -manifold $Z$ which must be homeomorphic to a connected sum of $S^{2} \times[0,1]$ and finitely many homotopy 3 -spheres. Since $H_{1}(\tilde{M})$ is zero, each of $\tilde{\Sigma}_{i}$ and $\tilde{\Sigma}_{i+1}$ separates $\tilde{M}$. Thus any translate of $\tilde{\Sigma}_{i}$ or $\tilde{\Sigma}_{i+1}$ in $Z$ separates $\tilde{\Sigma}_{i}$ from $\tilde{\Sigma}_{i+1}$ (since $\tilde{\Sigma}_{i}, \tilde{\Sigma}_{i+1}$ and their translates are essential). In $Z$, there is a $Z^{\prime}$ which is a connected sum of $S^{2} \times I$ and homotopy 3-spheres, which is bounded by a translate of $\tilde{\Sigma}_{i}$ and a translate of $\tilde{\Sigma}_{i+1}$ and whose interior contains no translates of $\tilde{\Sigma}_{i}$ or $\tilde{\Sigma}_{i+1}$. The submanifold of $M$ bounded by $\Sigma_{i}$ and $\Sigma_{i+1}$ is covered once or twice by $Z^{\prime}$, depending on whether $\Sigma_{i}$ is a 2-sphere or projective plane. This completes the proof.

(4.9) ExAmple. We give an example of a map from $S^{1}$ to a 3 -manifold that is compactly trivial (even if the map is extended to one rel a base ray by wedging $S^{1}$ with $[0, \infty)$ ) but is not properly trivial. This example fills in several gaps in the previous narrative. It shows that the homomorphism $\delta_{1}^{*}$ of (2.13) need not have trivial kernel (see (3.4) and following remarks). By (2.20) this then gives an example where (2.4) fails to be exact at $\pi_{1}(X)$ (see Example (2.22) and concluding remarks). Lastly, (3.9) shows that the domain of $\delta_{i}^{*}$ is trivial whenever the range of $\delta_{i}^{*}$ is trivial. Thus the obvious approach to building a homomorphism $\delta_{i}^{*}$ with nontrivial kernel is ruled out. This example shows that $\operatorname{ker} \delta_{i}^{*}$ can be nontrivial in spite of this restriction. Since $\operatorname{ker} \delta_{1}^{*}$ is contained in $\operatorname{ker} \varepsilon_{1}$, we also have by (3.8)(c) a space which admits compactly trivial but not properly trivial maps of $E^{1}$. This is no surprise since $S^{1}$ wedge $[0, \infty)$ is a carrier for an obvious map of $E^{1}$ whose proper triviality would imply the proper triviality of the map on $S^{1}$. After this example we will give a more compelling example for maps of $E^{1}$.

Let $T_{1} \subseteq T_{2}$ be a pair of solid tori arranged as shown in Figure 1. Embed $T_{2}$ in a solid torus $T_{3}$ so the pair $\left(T_{3}, T_{2}\right)$ is homeomorphic to the pair $\left(T_{2}, T_{1}\right)$. Continue, embedding $T_{i}$ in $T_{i+1}$ so $\left(T_{i+1}, T_{i}\right)$ is homeomorphic to $\left(T_{2}, T_{1}\right)$. Let $M=\cup^{\infty} T_{i}$. The inclusions $T_{i} \subseteq T_{i+1}$ and $T_{i} \subseteq M$ are homotopy equivalences. Let $J$ be the centerline 


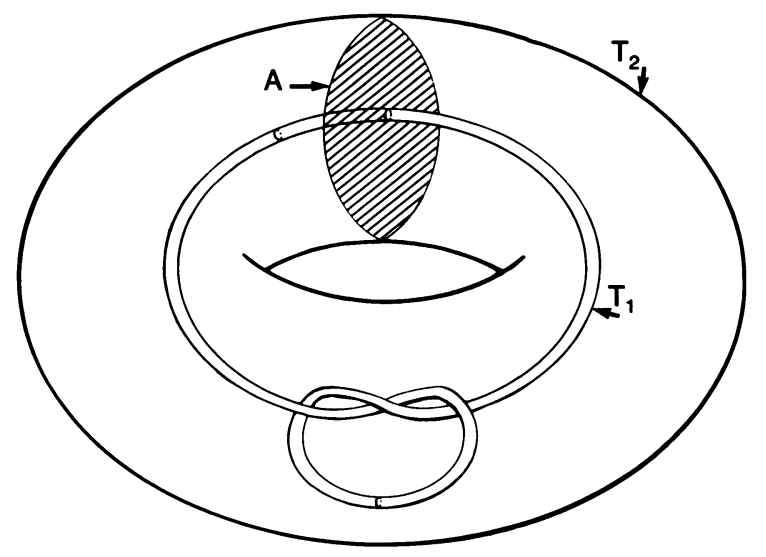

FIGURE 1

of $T_{1}$ and let $r:[0, \infty) \rightarrow M$ be proper so that $r^{-1}(J)=\{0\}$. We consider the inclusion of $J$ into $M$ wedged with $r$. Call this combined map $f$. It is compactly trivial because $M$ is a union of solid tori and the domain of $f$ is 1-dimensional. We will now show that the inclusion $J \subseteq M$ by itself is not properly trivial. We record some facts. We learned of the first from Joe Martin.

(4.10) There is a self-homeomorphism of $\left(T_{2}-\stackrel{\circ}{T}_{1}\right)$ that switches $\partial T_{1}$ and $\partial T_{2}$.

Proof. Split $\left(T_{2}-\stackrel{\circ}{T}_{1}\right)$ along the annulus $A$ in Figure 1 to yield a compact space $X$ bounded by a torus containing two copies $A^{+}$and $A^{-}$of $A$. An isotopy of $\partial X$ switches $A^{+}$and $A^{-}$. This isotopy gives a self-homeomorphism of a collar of $\partial X$ in $X$ which is fixed on the inner boundary of the collar and which is the end map of the isotopy on $\partial X$. Use the identity map off the collar to extend to all of $X$. Now resew along $A^{+}$and $A^{-}$to regain $T_{2}-\check{T}_{1}$.

(4.11) The surface $\partial T_{1}$ is incompressible in $T_{2}-\stackrel{\circ}{T}_{1}$.

Proof. Let Figure 1 picture an embedding of $T_{2}$ in $E^{3}$. The surface $\partial T_{1}$ is incompressible in $E^{3}-\stackrel{\circ}{T}_{1}$.

(4.12) The surface $\partial T_{2}$ is incompressible in $T_{2}-\stackrel{\circ}{T}_{1}$.

Proof. From (4.10) and (4.11).

(4.13) There is no map $h: S^{1} \times I \rightarrow T_{2}-\stackrel{\circ}{T}_{1}$ with $h^{-1}\left(\partial T_{1}\right)=S^{1} \times\{0\}, h^{-1}\left(\partial T_{2}\right)$ $=S^{1} \times\{1\}$ and $h \mid S^{1} \times\{0\}$ a generator of $\pi_{1}\left(T_{1}\right)$.

Proof. Assume such an $h$. Since $T_{1} \subseteq T_{2}$ is a homotopy equivalence, $h \mid S^{1} \times\{1\}$ is a generator of $\pi_{1}\left(T_{2}\right)$. A homotopy of $h$ supported on $S^{1} \times\left[\frac{1}{2}, 1\right]$ converts $h\left(S^{1} \times\{1\}\right)$ to a simple closed curve in $\partial T_{2}$ piercing $A$ exactly once. Cutting $T_{2}$ along a meridinal disk containing $A$, twisting, resewing and re-embedding in $E^{3}$ takes this curve to one bounding a disk in $E^{3}-\stackrel{\circ}{T}_{2}$ while taking $T_{1}$ back to itself. This would compress $\partial T_{1}$ in $E^{3}-\stackrel{\circ}{T}_{1}$.

(4.14) For all $i, \partial T_{i}$ is incompressible in $M-T_{1}$.

This follows from (4.11) and (4.12).

(4.15) The inclusion of $J$ into $M$ is not properly trivial. 


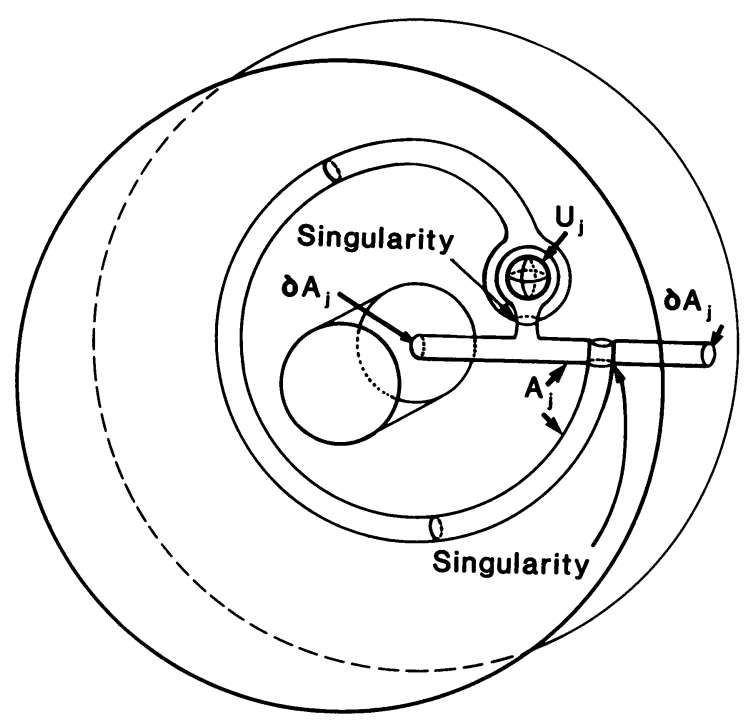

FIgURE 2. Section of $T_{j+1}-\stackrel{\circ}{T}_{j}$ near $E \times\{1\}$

Proof. Assume a proper $h: J \times[0, \infty) \rightarrow M$ with $h(x, 0)=x$ for all $x$ in $J$. Then $h^{-1}\left(T_{1}\right)$ is contained in some $J \times[0, n], h(J \times[0, n])$ is contained in the interior of some $T_{i}$, and there is an essential annulus $R$ in $J \times[n, \infty)$ with one boundary curve a component of $h^{-1}\left(\partial T_{i}\right)$, the other boundary curve a component of $h^{-1}\left(\partial T_{i+1}\right)$, and with all components of $h^{-1}\left(\partial T_{i} \cup \partial T_{i+1}\right)$ in $R$ trivial in $R$. Since $h(R)$ misses $T_{1}$ and since $\partial T_{i}$ and $\partial T_{i+1}$ are incompressible in $M-T_{1}$, we can alter $h$ on $\stackrel{\circ}{R}$ so that $h^{-1}\left(\partial T_{i} \cup \partial T_{i+1}\right)=\partial R$. Since $R$ is essential in $J \times[n, \infty)$, and $J$ is a generator of $\pi_{1}(M)$ and thus $\pi_{1}\left(T_{i}\right)$, the component of $\partial R$ mapping to $\partial T_{i}$ generates $\pi_{1}\left(T_{i}\right)$. The altered $h$ on $R$ now violates (4.13).

(4.16) ExAMPLE. Let $W$ be the Whitehead manifold. It is a contractible, open 3-manifold that is an ascending union of solid tori and it is described in [NW]. (It was first described in [W].) In [NW] a system of neighborhoods of the end of $W$ is given, and at the end of [NW] sufficient information is given about $\pi_{1}$ of these neighborhoods to show that the inverse sequence of $\pi_{1}$ of these neighborhoods is not semistable. Since $\pi_{1}(W)$ is trivial, the sequence $\left(\operatorname{ker} \beta_{1}\right)$ (notation $\left.\S 2\right)$ is exactly the $\pi_{1}$ sequence and $\lim ^{1}\left(\operatorname{ker} \beta_{1}\right)=\operatorname{ker} \varepsilon_{1}$ is not trivial (see (2.10)). Thus by (3.8)(c) there are compactly trivial proper maps of $E^{1}$ into $W$ that are not properly trivial. Since $W$ is an ascending union of solid tori, every proper map of $E^{1}$ into $W$ is compactly trivial. Since $\pi_{i}(W)$ is trivial for all $i$ every map of $S^{i}$ into $W$ proper rel any ray is properly trivial.

(4.17) EXAMPLE. This will be an example of a proper map from $E^{2}$ to a 3-manifold that is compactly trivial but not properly trivial. It should be compared to the hypotheses of (4.7). Let

$$
E=\{x \in \mathbf{C} \mid\|x\|<1\} \quad \text { and } \quad S^{1}=\{x \in \mathbf{C} \mid\|x\|=1\}
$$




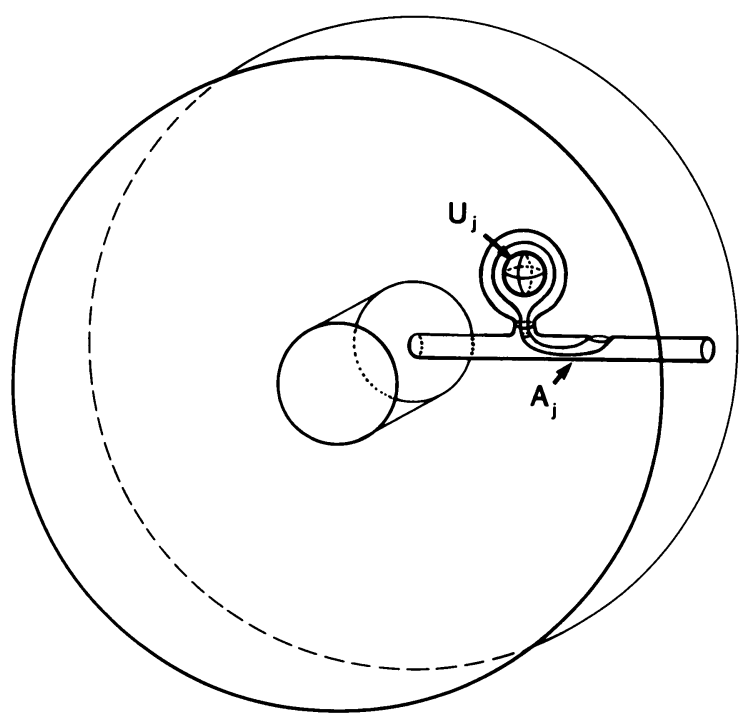

Figure 3. Section of $T_{j+1}-\stackrel{\circ}{T}_{j}$ near $E \times\{1\}$

be the open unit disk and the unit circle in the complex plane. Let

$$
p_{j}=\left(1-1 / 2^{2 j}, 1\right) \in E \times S^{1}, \quad 1 \leqslant j<\infty,
$$

be a sequence of points in the open solid torus. We can write $E \times S_{1}=\cup T_{j}$, where

$$
T_{j}=\left\{x \in \mathbf{C} \mid\|x\| \leqslant 1-1 / 2^{2 j-1}\right\} \times S^{1}, \quad 1 \leqslant j<\infty .
$$

For each $j \geqslant 1$, the layer $T_{j+1}-T_{j}$ contains $p_{j}$. Let $U_{j}$ be a round, open ball around $p_{j}$ whose closure lies in $\stackrel{\circ}{T+1}_{j+1}-T_{j}$. Let

$$
M=\left(E \times S^{1}\right)-\cup\left(U_{j}\right)
$$

For each $j \geqslant 1$ map the annulus

$$
A_{j}=\{x \in \mathbf{C} \mid j \leqslant\|x\| \leqslant j+1\}
$$

into $T_{j+1}-\stackrel{\circ}{T}_{j}$ as shown in Figure 2 and map $\bar{E} \subseteq \mathrm{C}$ into $\partial T_{1}$ so as to extend the map of $S^{1} \subseteq A_{1}$ into $\partial T_{1}$.

Sewing these maps together gives a singular proper map of $\mathrm{C} \cong E^{2}$ into $M$. Since the map on the annulus $A_{j}$ can be homotoped rel $\partial A_{j}$ in $T_{j}-\cup\left(U_{i}\right)$ to the picture in Figure 3 , the map is compactly trivial. If the map is properly trivial, then an extension to a proper map $g: E_{+}^{3} \rightarrow M$ exists. Half-balls $B_{1}$ and $B_{2}$ in $E_{+}^{3}$ centered at the origin exist so that $g^{-1}\left(\{0\} \times S^{1}\right)$ lies in $\stackrel{\circ}{B}_{1}, g\left(B_{1}\right)$ lies in some $\stackrel{\circ}{T}_{k}$, and $g^{-1}\left(T_{k+1}\right)$ lies in $\stackrel{\circ}{2}_{2}$. The ball $B_{3}=B_{2}-\stackrel{\circ}{B}_{1}$ maps into $M-\left(\{0\} \times S^{1}\right)$. The boundary of $B_{3}$ contains the annulus $A_{k}=\{x \in \mathbf{C} \mid k \leqslant\|x\| \leqslant k+1\}$, and $\partial B_{3}-\AA_{k}$ consists of two disks $D_{1}$ and $D_{2}$ where $D_{1}$ intersects $\partial B_{1}$ and $D_{2}$ does not. We have $g \mid A_{k}$ is the map on $A_{k}$ indicated by Figure $2, g\left(D_{1}\right) \subseteq \stackrel{\circ}{T}_{k}$, and $g\left(D_{2}\right) \subseteq M-T_{k+1}$. Let

$$
r: M-\left(\{0\} \times S^{1}\right) \rightarrow\left(T_{k+1}-\stackrel{\circ}{T}_{k}\right)-U_{k}
$$


be a retraction. We have $r g\left|A_{k}=g\right| A_{k}, g\left(D_{1}\right) \subseteq \partial T_{k}$, and $g\left(D_{2}\right) \subseteq \partial T_{k+1}$. Since tori are aspherical, we can homotope $r g$ in $\left(T_{k+1}-\stackrel{\circ}{T}_{k}\right)-U_{k}$ to a map agreeing with $g$ on $A_{k}$ and which carries $D_{1}$ and $D_{2}$ into the disks on $\partial T_{k+1}$ and $\partial T_{k}$ bounded by $\operatorname{Im} \partial A_{k}$. The map on $\partial B_{3}$ is trivialized in $\left(T_{k+1}-\stackrel{\circ}{T}_{k}\right)-U_{k}$ by the map on $B_{3}$. But a lift to the universal cover of $\left(T_{k+1}-\stackrel{\circ}{T}_{k}\right)-U_{k}$ shows that this is not possible. Thus the original map of $\mathbf{C} \rightarrow M$ is not properly trivial.

\section{REFERENCES}

[BK] A. K. Bousfield and D. M. Kan, Homotopy limits, completions and localizations, Lecture Notes in Math., vol. 304, Springer-Verlag, New York, 1972.

[B1] E. M. Brown, Proper homotopy theory in simplicial complexes, Topology Conf. (Virginia Polytechnic Institute and State University; Dickman and Fletcher, eds.), Lecture Notes in Math., vol. 375, SpringerVerlag, New York, 1974, pp. 41-46.

[B2] __ On the proper homotopy type of simplicial complexes, preprint.

[BF] E. M. Brown and C. D. Feustel, On properly embedding planes in arbitrary 3-manifolds, Proc. Amer. Math. Soc. (to appear).

[Ce] Z. Cerrin, On various relative proper homotopy groups, Tsukuba J. Math. 4 (1980), 177-202.

[Ch] T. A. Chapman, On some applications of infinite-dimensional manifolds to the theory of shape, Fund. Math. 76 (1972), 181-193.

[D] A. Z. Dymov, On the behavior at infinity of the fundamental group of a homologically trivial manifold, Math. USSR-Izv. 11 (1977), 529-550.

[EH] D. A. Edwards and H. M. Hastings, Čech and Steenrod homotopy theories with applications to geometric topology, Lecture Notes in Math., vol. 542, Springer-Verlag, New York, 1976.

[E] D. B. A. Epstein, The degree of a map, Proc. London Math. Soc. (3) 16 (1966), 369-383.

[F] S. Ferry, A stable converse to the Vietoris-Smale theorem with applications to shape theory, Trans. Amer. Math. Soc. 261 (1980), 369-386.

[Ge] R. Geoghegan, $A$ note on the vanishing of $\lim ^{1}$, J. Pure Appl. Algebra 17 (1980), 113-116; Corregendum 18 (1980), 231.

[Gr] B. I. Gray, Spaces of the same n-type for all $n$, Topology 5 (1966), 241-243.

[H] J. Hempel, 3-manifolds, Ann. of Math. Studies, vol. 86, Princeton Univ. Press, Princeton, N. J., 1976.

[HS] P. J. Hilton and U. Stammbach, $A$ course in homological algebra, Graduate Texts in Math., No. 4, Springer-Verlag, New York, 1971.

[KO] Y. Kodama and J. Ono, On fine shape theory. I and II, Fund. Math. 100 (1979), 29-39; 108 (1980), 89-98.

[L] F. Laudenbach, Topologie de la dimension trois, homotopie et isotopie, Astérisque, Vol. 12, Société Math. de France, 1974.

[Mac] S. Mac Lane, Homologv, Springer-Verlag, New York, 1975.

[Mas] W. S. Massey, Homologv and cohomology theory, Marcel Dekker, New York, 1978.

[Mih] M. L. Mihalik, Semistability at the end of a group extension, Trans. Amer. Math. Soc. 277 (1983), 307-321.

[Mil1] J. Milnor, On the Steenrod homologv theory, Mimeographed notes, Berkeley, 1960.

[Mil2] _ On axiomatic homologv theory, Pacific J. Math. 12 (1962), 337-341.

[NW] M. H. A. Newman and J. H. C. Whitehead, On the group of a certain linkage, Quart. J. Math. (Oxford) 8 (1937), 14-21.

[Q1] J. B. Quigley, An exact sequence from the $n$th to the $(n-1)$ st fundamental group, Fund. Math. 77 (1973), 195-210.

[Q2] __ Equivalence of fundamental approaching groups of movable pointed compacta, Fund. Math. 91 (1976), 73-83.

[S] N. E. Steenrod, Regular cycles of compact metric spaces, Ann. of Math. (2) 41 (1940), 833-851.

[W] J. H. C. Whitehead, A certain open manifold whose group is unity, Quart. J. Math. (Oxford) 6 (1935), $268-279$.

Department of Mathematics, State University of New York. Binghamton, New York 13901

Department of Mathematics, Southwest Texas State. University, San Marcos, Texas 78666 